\title{
Editorial
}

\section{Dinosaurs, Methicillin-Resistant Staphylococcus aureus, and Infection Control Personnel: Survival Through Translating Science Into Prevention}

\author{
William R. Jarvis, MD; Belinda Ostrowsky, MD, MPH
}

Some believe that dinosaurs became extinct because they failed to learn from their experiences and evolve in a changing world. The infection control community currently is witnessing an evolution in the epidemiology of methicillin-resistant Staphylococcus aureus (MRSA). How the infection control (and infectious diseases) community responds to this evolution will illustrate what it has learned from past science and experiences, whether it is capable of translating the science into prevention, and whether it will be successful ultimately in controlling antimicrobial-resistant pathogens in healthcare settings and the community. In other words, will the members of the infection control community become dinosaurs and irrelevant or will they apply the science in applied public health prevention and reverse the inexorable upward secular trends seen for many antimicrobial-resistant pathogens in healthcare settings?

If one conducts a Medline search of articles on $S$. aureus or MRSA, one will find that more than 20,000 have been written since 1966. Although we did not read each and every one of these articles, a quick review shows that a great deal is known about the science of $S$. aureus, the organisms, the microbiology, the mechanisms of resistance, typing systems, clinical features, epidemiology, risk factors, transmission, and prevention. Different typing systems (eg, serologic, phage, lysotyping, plasmid, enzyme, pulsed-field gel electrophoresis, and protein A) come and go. There is a continuing change in the antimicrobial susceptibility as new antimicrobials are introduced. Remember when quinolones and macrolides were first introduced and were going to be the next great drugs to eliminate MRSA? Obviously, understanding the biology of
MRSA is essential if efforts to develop a vaccine are to be successful, but more about that later.

It also is apparent that much more effort (as well as time and money) has been spent on studying the microbiologic characteristics of this organism than on applying the well-known principles of prevention (at least in healthcare settings). In reviewing the history of MRSA, we see its initial emergence in Europe (primarily in healthcare settings) and then its spread throughout the world. Although MRSA was identified in U.S. healthcare settings in the mid to late 1960 s, it did not become prevalent until the early 1980 s. In 1980 , data from the National Nosocomial Infections Surveillance (NNIS) System of the Centers for Disease Control and Prevention (CDC) showed that MRSA was emerging in only 4 hospitals, all of which were large teaching hospitals in the Northeast. ${ }^{1}$

During the past 20 years, we have seen MRSA move from large teaching hospitals to small teaching hospitals and from large community hospitals to small community hospitals (and then to long-term-care facilities) and become endemic in many, if not most, U.S. healthcare facilities. ${ }^{2,3}$ Currently, more than $50 \%$ of the strains of $S$. aureus causing hospital-acquired infections are methicillin resistant.2,3 A recent study by Richet et al. shows that the emergence of MRSA in healthcare settings throughout the world largely was due to the lack of understanding or implementation of effective MRSA infection control programs in hospitals, until MRSA had become endemic. ${ }^{4}$ Thus, although the science on prevention exists, ${ }^{5,6}$ it has not been put into practice.

In this issue of Infection Control and Hospital

Dr. Jarvis is from the Office of Extramural Research, National Center for Infectious Diseases, Centers for Disease Control and Prevention, Atlanta, Georgia. Dr. Ostrowsky is a former Epidemiology Intelligence Service Officer, Centers for Disease Control and Prevention, Atlanta, Georgia, and is currently at the Westchester County Department of Health, Westchester, New York.

Address reprint requests to Belinda Ostrowsky, MD, MPH, Westchester County Department of Health, 145 Huguenot St., 7th Floor, New Rochelle, NY 10801 
Epidemiology, multiple articles describe the changing epidemiology of MRSA in the United States. These articles illustrate the increasingly complex nature of both the microbiology and the epidemiology of MRSA and the increasing challenges we will have if we are to control this pathogen (and other antimicrobial-resistant pathogens). In the first article, by Baggett et al., ${ }^{7}$ we get a glimpse into the epidemiology of MRSA transmission among Alaska Natives. In Southwestern Alaska, more than $80 \%$ of $S$. aureus cultured from this population was methicillin resistant. Most of the infections were of the skin or soft tissues and $77 \%$ of the infections were thought to be community acquired. These patients were more likely to have received antimicrobials in the prior 180 days, but not to have five specific healthcare exposures. The authors conclude that the community-onset MRSA was not that of the hospital strain, but rather was true community acquisition. Although this is likely true, the exposure history was retrieved retrospectively from the medical records and may not have captured all hospital exposures (eg, a household member with repeated hospital exposures).

There are several potential biases in the study. First, the source of the "cases" was the medical center's electronic records system (looking for those with visits for skin infection) and the microbiology laboratory records of positive $S$. aureus cultures; thus, it is not surprising that most of the cases had skin or soft tissue infections. Second, it appears that the only MRSA isolates available for study were obtained in the period after the record review, so the identified cases and case isolates are from different individuals and time periods. Third, the MRSA isolates were examined for the mecA gene by polymerase chain reaction, but were not evaluated for the staphylococcal cassette chromosome mec (SCCmec). In contrast to other studies, the antimicrobial susceptibility patterns of community-acquired and non-community-acquired MRSA skin isolates were not different. Nevertheless, this article documents that MRSA is being transmitted in Alaska as it is in several locations and populations in the lower 48 states. ${ }^{810}$ Additional studies of the epidemiology of MRSA in these populations are urgently needed to identify cultural or other related practices (eg, crowded housing, antimicrobial use, or spa or hot tub exposures with the sharing of towels) to which prevention interventions could be targeted to slow or eliminate MRSA transmission.

In the second article in this issue of Infection Control and Hospital Epidemiology, Seal et al. ${ }^{11}$ examine the secular trend in antimicrobial resistance in $S$. aureus at the University of Chicago Hospitals during a 15-year period from 1986 to 2000 . Of the 17,287 S. aureus isolates available, the total annual number of isolates and the percent from outpatients ( $27 \%$ to $46 \%$ ) significantly increased. The annual rate of methicillin resistance increased significantly from $13 \%$ to $28 \%$, and this increase was seen in both the inpatient and the outpatient settings. The rate of non-betalactam resistance was significantly higher in MRSA isolates

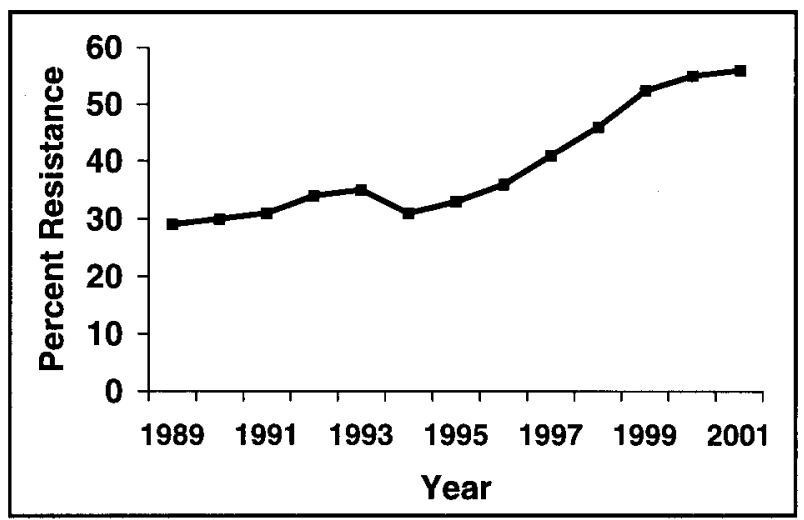

FIGURE. Proportion of Staphylococcus aureus nosocomial infections resistant to oxacillin in the intensive care unit. (National Nosocomial Infections Surveillance System data. Figure courtesy of S. Fridkin, Division of Quality Health Care Promotion, Centers for Disease Control and Prevention, Atlanta, GA.)

than in methicillin-susceptible $S$. aureus (MSSA) isolates. The increasing prevalence of MRSA is consistent with what is seen nationally at NNIS System hospitals, which reached a prevalence of methicillin resistance of $34 \%$ among $S$. aureus infections in 1995 and have now exceeded 50\% (many hospitals would thus be happy with rates of methicillin resistance of $13 \%$ to $28 \%$ [Figure]).$^{2,3,12}$ Nevertheless, the fact that the increasing rate of resistance has not plateaued suggests that if aggressive interventions are not undertaken to prevent transmission, increases in the prevalence of MRSA (and other antimicrobial-resistant pathogens) will continue.

In the third article, Jernigan et al. ${ }^{13}$ assess the prevalence of and risk factors for MRSA colonization at the time of admission to an Atlanta, Georgia, hospital. At this tertiary-care center, MRSA was isolated from 26 (2.7\%) of 974 patients presenting for admission. A questionnaire completed by all 26 of these patients (before the results of cultures were known by the investigators) revealed that they had been admitted to a healthcare facility in the preceding year, had at least one chronic disease, or both. When patients who were colonized with MRSA were compared with patients who were not colonized with MRSA, admission to a nursing home or a hospitalization of 5 days or longer during the preceding year were risk factors for MRSA colonization.

This study illustrates several important points. First, the epidemiology of community-onset or community-acquired MRSA varies from region to region and from city to city. One needs to evaluate the prevalence of such strains in the local community to better understand the epidemiology and dynamics of MRSA in that community. Second, studies that arbitrarily decide that MRSA recovered from a patient within 48 hours of admission must be due to community acquisition may misclassify patients who have had frequent healthcare exposures and who may have acquired MRSA during a previous hospitalization or healthcare exposure (eg, clinic visit) but were not 
detected because a culture was not performed until readmission. As suggested by Friedman et al. from Duke University, ${ }^{14}$ it may be best to categorize MRSA as healthcare associated, which includes those with hospital-acquired MRSA and those with community onset of healthcare-associated MRSA among those with healthcare exposures in the past 6 to 12 months, and those with true community-acquired MRSA (in those without healthcare exposures). At the least, this article suggests that studies in which community-acquired MRSA has been defined by medical record or microbiology review (of those with positive MRSA cultures in the first 48 hours after admission) alone without patient interview may be misclassifying those patients with previous hospital-acquired or healthcare-associated MRSA as having community acquisition. Others may wish to conduct such surveys to define the prevalence of MRSA at the time of patient admission and to determine the extent of true community-acquired MRSA among their patients.

In the fourth article in this issue of Infection Control and Hospital Epidemiology, by Fishbain et al.,15 prospective surveillance cultures of the nares and axillae for MRSA were performed at admission and discharge for patients admitted to the medicine, surgery, and pediatric wards and intensive care units at one hospital. Of 535 admission and 374 discharge cultures, 20 (3.7\%) of the patients were colonized with MRSA on admission and $6(1.7 \%)$ of those susceptible acquired MRSA during hospitalization. Those who were positive for MRSA on admission were more likely to be older, to have received antimicrobials within the previous year, to have been hospitalized within the previous 3 years, or to have a known history of MRSA. Those acquiring MRSA during hospitalization had longer hospitalizations than did those not acquiring MRSA and their six MRSA isolates had four pulsed-field gel electrophoresis patterns; this could mean either that some cases were not acquired in the hospital or that multiple strains were spreading simultaneously in the hospital. The authors conclude that surveillance cultures for all patients admitted or selective screening of those with risk factors and contact precautions for colonized patients may help to prevent MRSA transmission and infection.

This study documents that patients admitted with MRSA colonization (from a previous healthcare encounter) are an unrecognized source of MRSA for hospital transmission and that surveillance cultures to identify and isolate these reservoirs can reduce the risk of hospital transmission. These findings are similar to that of a previous study. ${ }^{16}$ Previously, Nicolle et al. ${ }^{17}$ documented that if only those patients with clinical cultures positive for MRSA were placed in isolation, the prevalence of MRSA continued to rise. When active surveillance cultures were implemented, the prevalence and incidence of MRSA were reduced. Subsequently, several studies have documented that active surveillance cultures for all or selected patients (based on the epidemiology of MRSA in one's hospital and community), in combination with placing these identified reservoirs in contact precautions, can dramatically reduce or eliminate the prevalence or incidence of MRSA. ${ }^{1821}$

Recently, the Society for Healthcare Epidemiology of America (SHEA) published a position paper endorsing the use of active surveillance cultures in combination with contact isolation (in conjunction with other pillars of infection control, education, hand hygiene, and judicious use of antimicrobials) for the control of MRSA and vancomycinresistant Enterococcus (VRE). ${ }^{22}$ The data support that this approach is effective in controlling MRSA and VRE transmission in healthcare settings. An additional reason to implement programs to control MRSA transmission in healthcare settings is illustrated in the fifth article in this issue of Infection Control and Hospital Epidemiology, by Calfee et $\mathrm{al}^{23}$ They assessed the frequency with which MRSA was spread from MRSA-colonized or MRSA-infected patients to their household and community contacts. MRSA subsequently was isolated from $14.5 \%$ of the contacts; close contact with an individual colonized or infected with MRSA placed the contact at 7.5-fold greater risk of becoming colonized with MRSA. Thus, hospital strains of MRSA can be introduced and spread in the community (again, such individuals would not be recognized in most studies as having healthcare-associated MRSA).

In the past several months, several reasons have been advanced for not wanting to try to control MRSA or VRE. These have included that it may be too difficult, too expensive, or too time-consuming or that those in the field "don't want to be bothered." As the epidemiology of MRSA evolves and the emergence of vancomycin intermediateresistant $S$. aureus and vancomycin-resistant $S$. aureus continues, ${ }^{24-26}$ the interruption of the transmission of MRSA in healthcare settings becomes more and more important. We have the science, now we need the will to implement the science into clinical practice for prevention. ${ }^{22}$

In the review article in this issue of Infection Control and Hospital Epidemiology, Said-Salim et al. ${ }^{27}$ discuss the molecular basis for the methicillin resistance of classic hospital versus community-acquired MRSA. In all MRSA, the methicillin resistance is encoded in the site-specific integration of a $26-$ to $67-\mathrm{kb}$ SCCmec element into the genome of methicillin-susceptible strains; this is a mobile element that contains the mecA gene, which encodes for altered low-affinity penicillin-binding protein $2 \mathrm{~A}$ and its regulators. ${ }^{28}$ Polymerase chain reaction assays have revealed that most hospital-acquired MRSA strains are one of three SCCmec types-I, II, or III. ${ }^{28}$ In contrast, most of the prototype community-acquired MRSA strains have belonged to SCCmec type IV. These include the four pediatric deaths caused by the mono-beta-lactam MRSA clone in Minnesota and North Dakota. ${ }^{29,30}$ Initially, the true community acquired (often with methicillin but not otherwise multidrug resistant) and the true hospital acquired (methicillin and otherwise multidrug resistant) were genetically distinct. However, in the past 6 to 12 months, there have been reports of hospital transmission of "true" community-acquired MRSA strains ${ }^{31}$ and community transmission of "true" hospital-acquired MRSA 
strains..$^{23}$ In the future, the genetic purity of separation of the hospital and community MRSA strains will diminish as such strains are transmitted in new settings.

Additional studies of the genetics of the MRSA strains circulating in our communities (health care and non-health care) will be useful. However, a review of Medline from 1966 to now shows the enormous amount that we have learned about $S$. aureus and MRSA that can be applied right now. During the past decade, we have seen a dramatic increase of MRSA infections in Native American populations and in jail and prison populations.810 The prevalence of MRSA (colonization and infection) is unknown in these populations, but is likely much higher than any of us suspect. Continued basic science will be critical for additional prevention efforts, such as vaccines. However, an $S$. aureus vaccine has been discussed for decades and the recent version of such a vaccine has had limited trials (only in populations that are at high risk), appears to have limited immunogenicity and duration of protection, and, to have longer-term protection, would require repeated administration. ${ }^{32}$

Until an effective vaccine or other intervention is available, the application of known prevention measures should be our highest priority. These should include the judicious use of antimicrobials, the reinforcement of hand hygien $e^{33}$ and other basic hygiene measures in atrisk populations (including not sharing towels and soap in prisons) in the community and in hospitals (including the CDC's Campaign to Prevent Antimicrobial Resistance in Healthcare Settings $\mathrm{s}^{34}$ ), and more active measures (eg, active surveillance cultures and contact isolation) ${ }^{22}$ These measures have been documented to reduce or eliminate MRSA transmission in healthcare facilities and do not require advanced genotyping capacity to accomplish. More specific recommendations for preventing community-acquired strains are difficult until the epidemiology in specific at-risk populations is defined. Most of the MRSA we need to control is healthcare related, as the current prevalence of MRSA in the community population is less than $1 \%$. Despite this low prevalence of MRSA in the general population, pulsedfield gel electrophoresis typing of isolates from selected populations has shown that most community-acquired isolates in a particular community are clonal, suggesting person-to-person transmission. ${ }^{35}$ The time to act is now, before the prevalence of MRSA in the community begins to rise and we end up with $50 \%$ of the community strains becoming methicillin resistant. Prevention should be our primary aim. If we implement what we know (as additional basic and clinical research is being conducted for future prevention interventions), maybe we will turn the tide on MRSA and other antimicrobial-resistant pathogens and not end up looking like dinosaurs on this topic.

\section{REFERENCES}

1. Haley R, Hightower A, Khabbaz R, et al. The emergence of methicillinresistant Staphylococcus aureus infections in United States hospitals: pos- sible role of the house staff-patient transfer circuit. Ann Intern Med 1982;973:297-308.

2. Centers for Disease Control and Prevention. National Nosocomial Infections Surveillance (NNIS) System report: data summary from January 1992 to June 2002, issued August 2002. Am I Infect Control 2002;30:458-475.

3. Centers for Disease Control and Prevention. National Nosocomial Infections Surveillance (NNIS) System report: data summary from January 1992-June 2001, issued August 2001. Am J Infect Control 2001;29:404-421.

4. Richet H, Benbachir M, Brown D, et al. Are there regional variations in the diagnosis, surveillance, and control of methicillin-resistant Staphylococcus aureus? Infect Control Hosp Epidemiol 2003;24:334-341.

5. Garner JS, Hospital Infection Control Practices Advisory Committee. Guideline for isolation precautions in hospitals. Infect Control Hosp Epidemiol 1996;17:53-80.

6. Centers for Disease Control and Prevention. Recommendations for preventing the spread of vancomycin resistance: recommendations of the Hospital Infection Control Practices Advisory Committee (HICPAC). MMWR 1995;44(RR12):1-13.

7. Baggett HC, Hennessy TW, Leman R, et al. An outbreak of community-onset methicillin-resistant Staphylococcus aureus skin infections in Southwestern Alaska. Infect Control Hospital Epidemiol 2003;24:397402.

8. Groom A, Wolsey D, Naimi T, et al. Community-acquired methicillinresistant Staphylococcus aureus in a rural American Indian community. JAMA 2001;286:1201-1205.

9. Anonymous. Outbreaks of community-associated methicillin-resistant Staphylococcus aureus skin infections: Los Angeles County, California, 2002-2003. MMWR 2003;52:88.

10. Anonymous. Methicillin-resistant Staphylococcus aureus skin or soft tissue infections in a state prison: Mississippi, 2000. JAMA 2002;287:181182.

11. Seal JB, Moreira B, Bethel CD, Daum RS. Antimicrobial resistance in Staphylococcus aureus at the University of Chicago Hospitals: a 15-year longitudinal assessment in a large university-based hospital. Infect Control Hospital Epidemiol 2003;24:403-408.

12. Fridkin SK, Gaynes RP. Antimicrobial resistance in intensive care units. Clinic Chest Med 1999;20:303-316.

13. Jernigan JA, Pullen AL, Flowers L, Bell M, Jarvis WR. Prevalence of and risk factors for colonization with methicillin-resistant Staphylococcus aureus at the time of hospital admission. Infect Control Hospital Epidemiol 2003;24:409-414.

14. Friedman ND, Kaye KS, Stout JE, et al. Health care-associated bloodstream infections in adults: a reason to change the accepted definition of community-acquired infections. Ann Intern Med 2002;137:791797.

15. Fishbain JT, Lee JC, Nguyen HD, et al. Nosocomial transmission of methicillin-resistant Staphylococcus aureus: a blinded study to establish baseline acquisition rates. Infect Control Hospital Epidemiol 2003;24:415421.

16. Jernigan JA, Titus MG, Groschel DH, Getchell-White S, Farr BM. Effectiveness of contact isolation during a hospital outbreak of methicillin-resistant Staphylococcus aureus. Am J Epidemiol 1996;143:496504.

17. Nicolle LE, Dyck B, Thompson G. Regional dissemination and control of epidemic methicillin-resistant Staphylococcus aureus: Manitoba Chapter of CHICA-Canada. Infect Control Hosp Epidemiol 1999;20:202205.

18. Farr BM, Salgado CD, Karchmer TB, Sherertz RJ. Can antibiotic-resistant nosocomial infections be controlled? Lancet Infect Dis 2001;1:3845.

19. Haley RW, Cushion NB, Tenover FC, et al. Eradication of endemic methicillin-resistant Staphylococcus aureus infections from a neonatal intensive care unit. I Infect Dis 1995;171:614-624.

20. Jans B, Suetens C, Struelens M. Decreasing MRSA rates in Belgian hospitals: results from the national surveillance network after introduction of national guidelines. Infect Control Hosp Epidemiol $2000 ; 21: 419$.

21. Struelens MJ, Ronveaux O, Jans B, Mertens R. Methicillin-resistant Staphylococcus aureus epidemiology and control in Belgian hospitals, 1991 to 1995: Groupement pour le Depistage, l'Etude et la Prevention des Infections Hospitalieres. Infect Control Hosp Epidemiol 1996;17:503508

22. Muto CA, Jernigan JA, Ostrowsky BE, et al. SHEA guideline for preventing nosocomial transmission of multidrug-resistant strains of Staphylococcus aureus and Enterococcus. Infect Control Hospital Epidemiol 2003;24:362-386.

23. Calfee DP, Durbin LJ, Germanson TP, Toney DM, Smith EB, Farr BM. 
Spread of methicillin-resistant Staphylococcus aureus (MRSA) among household contacts of individuals with nosocomially acquired MRSA. Infect Control Hospital Epidemiol 2003;24:422-426.

24. Centers for Disease Control and Prevention. Staphylococcus aureus with reduced susceptibility to vancomycin: Illinois, 1999. MMWR 2000;48:1165-1167.

25. Smith TL, Pearson ML, Wilcox KR, et al. Emergence of vancomycin resistance in Staphylococcus aureus. N Engl J Med 1999;340:493-501.

26. Centers for Disease Control and Prevention. Public Health Dispatch: vancomycin-resistant Staphylococcus aureus. Pennsylvania, 2002. MMWR 2002;51:902.

27. Saïd-Salim B, Mathema B, Kreiswirth BN. Community-acquired methicillin-resistant Staphylococcus aureus: an emerging pathogen. Infect Control Hospital Epidemiol 2003;24:451-455.

28. Ito T, Katayama Y, Asada K, et al. Structural comparison of three types of staphylococcal cassette chromosome mec integrated in the chromosome in methicillin-resistant Staphylococcus aureus. Antimicrob Agents Chemother 2001;45:1323-1336.

29. Herold BC, Immergluck LC, Maranan MC, et al. Community-acquired methicillin-resistant Staphylococcus aureus in children with no identified predisposing risk. JAMA 1998;279:593-598.

30. Salgado CD, Farr BM, Calfee DP. Community-acquired methicillin-resistant Staphylococcus aureus: a meta-analysis of prevalence and risk fac- tors. Clin Infect Dis 2003;36:131-139.

31. Graham PL III, O'Keefe M, Saiman L. Risk factors for healthcareassociated acquisition of community-acquired methicillin-resistant Staphylococcus aureus (CA-MRSA) in post-partum women. Presented at the 13th Annual Scientific Meeting of the Society for Healthcare Epidemiology of America; April 5-8, 2003; Arlington, VA. Abstract 153.

32. Shinefield H, Black S, Fattom A et al. Use of a Staphylococcus aureus conjugate vaccine in patients receiving hemodialysis. $N$ Engl $J$ Med 2002;346:491-496.

33. Centers for Disease Control and Prevention. Guideline for hand hygiene in health-care settings: recommendations of the Healthcare Infection Control Practices Advisory Committee and the HICPAC/SHEA/APIC/IDSA Hand Hygiene Task Force. MMWR 2002;51:1-45

34. Centers for Disease Control and Prevention. Campaign to Prevent Antimicrobial Resistance in Healthcare Settings: 12-Steps for Healthcare Settings. Atlanta, GA: Centers for Disease Control and Prevention; 2002. Available at www.cdc.gov/drugresistance/healthcare/default.htm.

35. Groom AV, Wolsey DH, Naimi TS, et al. Community-acquired methicillin-resistant Staphylococcus aureus in a rural American Indian community. JAMA 2001;286:1201-1205. 